Nonlinear analysis and design of an active multiplier

antenna array

Shin-Der Yang and Tah-Hsiung Chu*

Electrical Engineering Department, National Taiwan University

No.1, Sec. 4, Roosevelt Road, Taipei, Taiwan, R.O.C.

\begin{abstract}
1. Introduction
Spatial and Quasi-optical power combining technique is a promising approach for overcoming the inherent power limitation of solid-state devices at the millimeter-wave frequency range [1]. Such array can effectively cooperate with large numbers of devices. By combining their power in free space, it may achieve low combining loss and hence high combining efficiency. However, at nillimeter and sub-millimeter wave frequency region solid-state devices have limited power performance with poor power added efficiency. In addition, there is always a frequency limit at which a solid-state oscillator can operate. In that limit, the negative resistance produced by active device can not compensate the resonator loss.

One way to generate signal at such frequency is to use the active device nonlinearity to yield with higher-order harmonics as a frequency multiplier. There are several method to increase the output harmonic power. In [2], the multiplier is designed with optimized harmonic termination impedance for higher output power. In [3], a periodic load method is proposed by strongly coupling between devices. However, strongly coupled oscillator array must encounter with problems such as multi-mode of node voltages and symmetric arrangement of oscillators. In this paper, we develop an active multiplier array structure which consists of oscillators by directly matching at the second-harmonic frequency and considering the clipping effect within FET. Using this structure, the multiplier antenna array not only can yield with higher output harmonic signal power but also has the benefit for scanning array application. The scanning angle under second harmonic operation is twice of that under fundamental operation [4]
\end{abstract}

II. Formulation

In the analysis, we firstly use the harmonic-balance method to solve the input impedance of individual oscillator and then apply the weakly coupled oscillator theory to 
analyze the phase distribution of antenna array. Assume the following two conditions are satisfied. One is the free-running oscillation frequency and amplitude deviation between array elements are small. In addition, the change of voltage amplitude of individual element after coupling is small [5]. One can then separate the amplitude and phase dynamic into individual differential equation. For the $\mathrm{i}$-th oscillator, its voltage waveform at coupling node is represented as

$$
V_{i}(t)=\operatorname{Re}\left[\left(A_{i, 0}+\Delta \widetilde{A}_{i}(t)\right) e^{j\left(2 \pi / i+\phi_{i}(t)\right)}\right]
$$

where $A_{i, n}$ is the $\mathrm{i}$-th oscillator free-running voltage amplitude at fundamental frequency. $\Delta \widetilde{A}_{i}(t)$ is the amplitude transient after inter-locking, and the reference frequency. $f_{r}$ is the injection source frequency or the average free-running frequency of entire oscillator array when no extern injected source presents. By only considering the weakly nonlinear oscillator operation, one can write the dynamic equation of phase and amplitude as

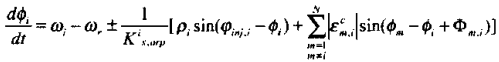

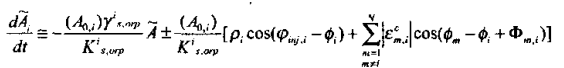

where

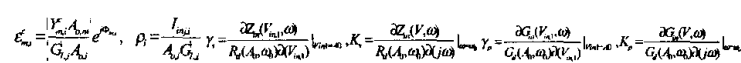
In (2) and (3), ' + ' sign is for parallel resonance oscillation, and '- sign is used for series resonance oscillation. $K$ and $\gamma$ are nonlinear parameters to be solved using the harmonic-balance method. From (2) and (3), one can see that phase of each array element can be varied by tuning the free-running frequency of each oscillator in the array.

\section{Design and measurement}

\section{A. Antenna element}

Figure 1 is the schematic diagram of each active multiplier antenna element. It uses source feedback configuration with $\mathrm{L} 1$ to give negative resistance at $4 \mathrm{GHz}$ and $\mathrm{L} 21+\mathrm{L} 22=$ $\lambda_{\mathrm{g}} / 4$ at $4 \mathrm{GHz}$. Thus, the input impedance at $B$ for $L 21+L 22$ is an open at $4 \mathrm{GHz}$ and a short at $8 \mathrm{GHz}$ The length of $\mathrm{L} 21$ is $7 \mathrm{~mm}$ and $\mathrm{L} 22$ is $4.7 \mathrm{~mm}$. From the node voltages that derived by initial design, we can vary the L22 length to match Zout and Zload at the second- harmonic frequency. Figure 2 shows Zout and Zload on the Smith chart for two different $L 22$. One can see that $L 22=2.4 \mathrm{~mm}$ has a better match than that in the initial design with $\mathrm{L} 22=4.7 \mathrm{~mm}$. The simulation and measurement results of the output second-harmonic signal power level is $3 \mathrm{dBm}$ for $L 22=4.7$ and $6 \mathrm{dBm}$ for $L 22=2.4 \mathrm{~mm}$. Furthermore, one can adjust the bias point to yield with a larger second-harmonic current. From Fig. 3, one can sec that the bias point with $\mathrm{Vgs}=0.2 \mathrm{~V}$ and $\mathrm{Vds}=3 \mathrm{~V}$ has a better clipping current waveform for the second -harmonic frequency. At this bias point, the second-harmonic output is 9 
$\mathrm{dBm}$ from simulation, which is close to the measurement result of $9.5 \mathrm{dBm}$.

\section{B. Multiplier antenna array}

For the active multiplier antenna array design, we use the neighbor coupling architecture. Table 1 gives the array design parameters. From (2), one can solve the fundamental and second-harmonic node voltage phase difference, and calculate the array radiation pattern after edge detuning. Table 2 gives the measured and simulated results of array EIRP, and Fig. 4 shows the measured and simulated array radiation pattern.

\begin{tabular}{|l|l|}
\hline array clements & 3 \\
\hline fundamental osc. frequency & $4 \mathrm{GHz}$ \\
\hline output frequency & $8 \mathrm{GHz}$ \\
\hline coupling factor $\left(\varepsilon=G_{i}^{\prime} / Y_{11}^{c}\right)$ & 0.2 \\
\hline antenna spacing & $0.45 \lambda$ \\
\hline substrate & Duroid $5880, \varepsilon,=2.2, \mathrm{hs}_{\mathrm{s}}=1.57 \mathrm{~mm}$ \\
\hline
\end{tabular}

Table 1 Design parameters of active multiplier antenna array.

\begin{tabular}{|c|c|c|}
\hline feedback line length & $\begin{array}{c}\text { measurement } \\
\text { result }\end{array}$ & $\begin{array}{c}\text { simulation } \\
\text { result }\end{array}$ \\
\hline $\mathrm{L} 22=4.7 \mathrm{mmn}, \mathrm{L} l=9.1 \mathrm{~mm}$ & $17.4 \mathrm{dBm}$ & $19 \mathrm{dBm}$ \\
\hline $\mathrm{L} 22=2.7 \mathrm{~mm}, \mathrm{~L}=8.5 \mathrm{~mm}$ & $21.5 \mathrm{dBm}$ & $22.68 \mathrm{dBm}$ \\
\hline
\end{tabular}

Table 2 Measurement and simulation results of antenna array EIRP

Summary

Analysis of a coupled active multiplier antenua array on its matching method for the second-harmonic output power is developed. By using the harmonic-balance method and weakly couple oscillator dynamic theory, one can well predict its second-harmonic output power, matching condition and phase distribution between array elements. Impedance matching using large-signal and small-signal analysis concept can increase the output power about $3 \mathrm{~dB}$. By further considering the clipping effect of bias condition, the output power can be increased about $6 \mathrm{~dB}$ to be $9.5 \mathrm{dBm}$. The measurement results are shown in good agreement with simulation results.

\section{References}

[1] K. Chang and C. Sun, "Millimeter-wave power-combining techniques", IEEE Trans. Microwave Theory Tech., vol. 31 no. 2, pp. 91-107, Feb. 1983.

[2] D. G. Thomas, Jr. and G. R. Branner, "Opcimization of active microwave frequency multiplier performance utilizing harmonic terminating impedance," IEEE Trans. Microwave Theory Tech., vol. 44, pp. 2617-2624, Dec. 1996.

[3] A. Mortazawi, H. D. Foltz, and T. Itoh, "A periodic second harmonic spatial power combining oscilfator", IEEE Trans. Microwave Theory Tech., vol. 40, pp. 851-856, May 1992.

[4] A. Alexnian, H. C. Chang, and R. A. York, "Enhanced scanning range in coupled oscillator arrays 
utilizing frequency multipliers," 1995 IEEE Antennas Propagation Symposium Dig. pp. 1308-1310, 1995.

[5] R. A. York, "Nonlinear Analysis of phase relationships in quasi-optical oscillator arrays," IEEE Trans. Microwave Theory Tech., vol. 41, pp. 1799-1809, Oct. 1993.

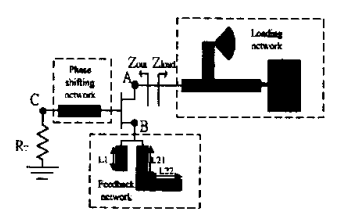

Fig.l Schematic diagram of active multiplier array element.
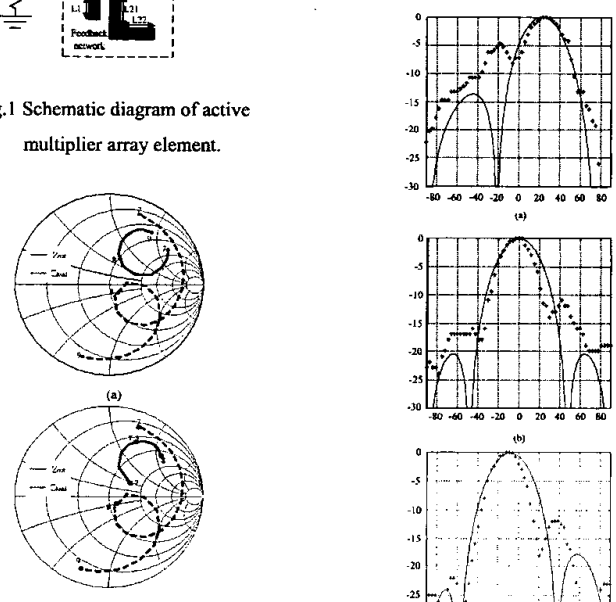

Fig. 2 The input impedance Zout and load impedance Zload at the secondharmonic frequency with (a) $L 22=2.4$ $\mathrm{mm}$ and (b) $\mathrm{L} 22=4.7 \mathrm{~mm}$
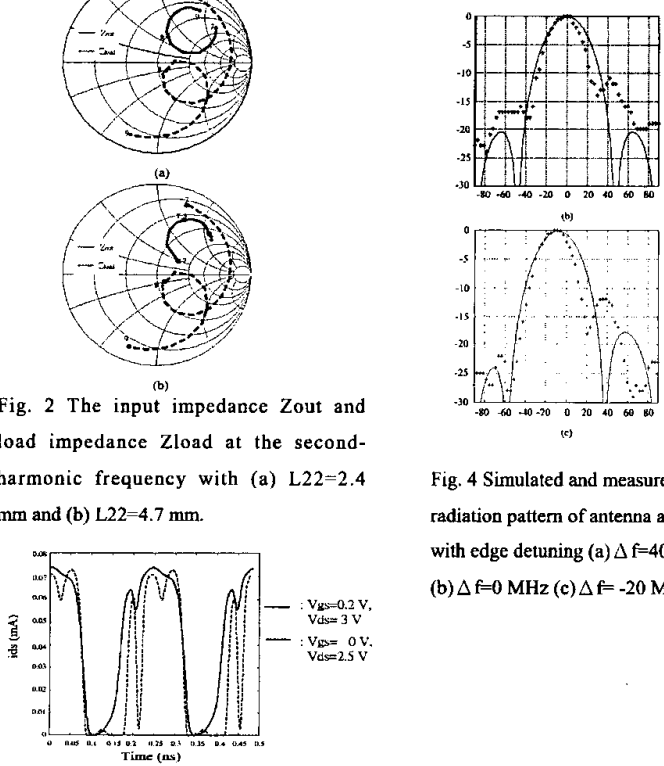

Fig. 4 Simulated and measured radiation pattern of antenna array with edge detuning (a) $\Delta \mathrm{f}=40 \mathrm{MHz}$ (b) $\Delta \mathrm{f}=0 \mathrm{MHz}$ (c) $\Delta \mathrm{f}=-20 \mathrm{MHz}$.

Fig. 3 Simulation result of the drain current ias waveform. 